\title{
Imaging of acute versus pathological pain in humans
}

\author{
J. Lorenz ${ }^{a, *}$, K.L. Casey ${ }^{b}$ \\ ${ }^{a}$ Institute of Neurophysiology and Pathophysiology, University Clinic Hamburg-Eppendorf, Martinistrasse 52, D-20246 Hamburg, Germany \\ b Department of Neurology, University of Michigan, Medical Center Drive, Ann Arbor, MI 48109, USA
}

Received 8 July 2004; accepted 12 July 2004

Available online 11 August 2004

\begin{abstract}
Pain subserves different functions. Acute pain from the intact body alerts the victim to immediately react and withdraw from the bodily threat, ideally before an injury happens. However, during manifest injury and tissue inflammation, withdrawal and flight are no longer adaptive. Instead, sparing the affected body part to promote healing requires heightened awareness and avoidance behaviour over longer periods of time. Quality and time scales of behavioural adaptations are therefore substantially different between pain during normal compared to abnormal tissue states. Given these functional differences we postulated that the two phenomena also recruit different forebrain systems. We used positron emission tomography (PET) and subtracted scans obtained during painful heating of normal skin from scans during equally intense but normally non-painful heating of capsaicin-treated skin. This comparison reveals the specific activation of a medial thalamic pathway to limbic forebrain structures such as anterior insula, perigenual anterior cingulate, ventral striatum, and prefrontal cortex during pain originating in the chemically sensitised skin. It is possible that the unique forebrain recruitment by pain under a patho-physiological tissue status is caused by a significantly greater facilitation of the multi-synaptic projections from the spino-parabrachial tract of the superficial dorsal horn to the medial thalamus compared to deeper and direct lateral thalamic projections from the spino-thalamic tract.

(C) 2004 European Federation of Chapters of the International Association for the Study of Pain. Published by Elsevier Ltd. All rights reserved.
\end{abstract}

\section{Introduction}

After the first generation of neuroimaging research of pain in humans demonstrated a reasonable consistency of brain areas that are activated by well-controlled experimental pain stimuli in normal subjects (for review see Casey, 1999), researchers in the field have started to use these methodologies to elucidate the mechanisms of abnormal pain states. Normally, if a stimulus is intense enough to activate nociceptors, multiple areas of the brain such as the thalamus, the primary (SI) and secondary (SII) somatosensory cortices, posterior and anterior parts of the insula, and the anterior cingulate cortex

\footnotetext{
${ }^{*}$ Corresponding author. Tel.: +49 4042803 3188; fax: +49 4042803 7752.

E-mail address: lorenz@uke.uni-hamburg.de (J. Lorenz).
}

(ACC) respond to this input in a correlated manner with perceived intensity (Coghill et al., 1999). Under conditions of specific psychological interventions such as hypnosis (Rainville et al., 1997), pain anticipation (Ploghaus et al., 1999), or placebo cognitions (Petrovic et al., 2002) some of these regions such as ACC and the anterior insula appear to subserve more specifically the attentional and affective pain processes (see also Sprenger et al., 2004). This aspect of the pain experience has long been distinguished from the sensory-discriminative component of pain (Melzack and Casey, 1968), which exhibits closer association to SI and SII of the parietal lobe. This latter assumption is supported by the somatotopic organisation of hemodynamic activity in these brain areas following nociceptive stimuli to different parts of the body (Andersson et al., 1997; Bingel et al., 2003). Other areas such as brainstem, basal ganglia, cerebellum 
and various structures within the prefrontal cortex yielded activation by experimental pain in several studies, their role in pain processing, however, remained elusive and is mainly deduced from their importance in other cognitive, motor or behavioural functions.

In pathological pain states, inflammation or nerve lesions can exaggerate the sensitivity to painful stimuli (hyperalgesia), or cause pain following warm or touch stimuli (allodynia). Evidence from spinal reactions during inflammatory hyperalgesia in animals suggests that central sensitization contribute significantly to these phenomena. We used positron emission tomography (PET) to address the question of whether nociceptive activity originating from sensitized skin in humans acts upon the same forebrain structures as under normal skin conditions. Unlike functional magnetic resonance imaging, PET allows monitoring of baseline changes in activity that may accompany an experimentally induced sensitized state.

Based on the fact that there are distinct populations of spinal projection neurons in the dorsal horn that differ in their supraspinal targets we hypothesized that they may play different roles in normal versus abnormal pain processes. Neurons located in the superficial layers Rexed's lamina I and outer II) belong mainly to the nociceptive-specific (NS) type and project within the spino-parabrachial tract (SpBT) to the lower brainstem, an important relais area to hypothalamic and limbic sites such as amygdala, medial thalamus and orbitofrontal cortex. Deeper projections from lamina V are mostly wide-dynamic-range (WDR) neurons that ascend within the spinothalamic tract (STT) to lateral thalamic nuclei from where they reach the somatosensory cortices (Hunt and Mantyh, 2001)). Although there is certainly overlap between the two projections systems, recent evidence indicates that only the SpBT fibers, which express the peptidergic NK-1 receptor, respond to electrical highfrequency stimulation (HFS) of C-fiber strength with long-term potentiation (LTP) (Ikeda et al., 2003). Identical HFS, in turn, induces neurogenic hyperalgesia and inflammatory skin reactions when applied to human test subjects (Klein et al., 2004; Sandkühler and Ruscheweyh, 2004). Given a particular importance of superficial SpBT neurons in mediating spinal sensitization during inflammation of their receptive fields, different supraspinal targets of these neurons than that of STT neurons should give rise to different neuroimaging results when C-fiber induced hyperalgesia and normal pain are compared with each other.

\section{Methods}

To test this hypothesis we applied topical capsaicin to induce C-fiber mediated hyperalgesia in 14 healthy male subjects and examined their responses to defined contact heat stimuli before and after treatment. Details are described in our original article (Lorenz et al., 2002). A critical aim of our experiment was to match subjective intensities of a slowly ramped and continuously applied contact heat stimulus across normal and sensitised skin conditions because we wanted to minimize a confound with perceived intensity. After establishing the appropriate capsaicin concentrations, duration of topical treatment and intensities of the contact heat probe in pilot tests outside the scanner we measured cerebral blood flow (CBF) reactions using PET after $\mathrm{O}^{15}$-water injections (Sprenger et al., 2004) and computed subtraction maps comparing trials of differently applied but equally perceived pain intensities of heat stimuli on normal (around $47{ }^{\circ} \mathrm{C}$ ) and treated (around $43^{\circ} \mathrm{C}$ ) skin.

\section{Results and discussion}

Results are given in Fig. 1. It illustrates that key structures of the lateral pain pathway, reached by the STT, such as the lateral thalamus and the SII/posterior insula region were in fact equally activated by the equally intense stimuli and therefore eliminated by the

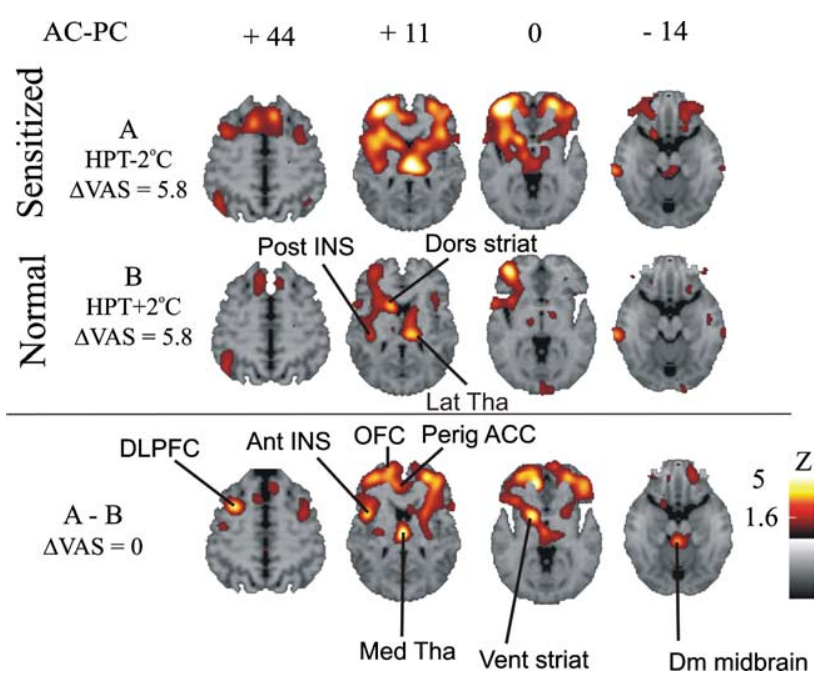

Fig. 1. Comparison of $\mathrm{O}^{15}$-water PET scans during equally painful contact heat stimuli elicited on capsaicin-treated (A) and normal (B) skin at the left forearm of 14 healthy volunteers. Rows A and B indicate the subtraction maps against rest condition with normal skin, bottom row illustrates the direct subtraction map A minus B. The four columns represent different horizontal slice levels relative to the line between the anterior and posterior commissure (AC-PC) indicated at the top. Note that areas such as posterior insula, dorsal striatum and lateral thalamus are equally activated by the two stimuli and therefore disappear in the direct comparison. In contrast, activity areas such as anterior insula, ventral striatum, orbitofrontal cortex (OFC) and dorsolateral prefrontal cortex (DLPFC), and medial thalamus are significantly more activated during sensitised than normal skin condition and appear in the direct comparison image (data from Lorenz et al., 2002). 
subtraction (bottom row). In contrast, key structures of the medial pain pathway (medial thalamus, perigenual ACC, anterior insula, ventral striatum, prefrontal cortex, and midbrain), that partly receive direct input from the SpBT, exhibited significantly more activity when pain was inflicted on the treated skin and induced by normally warm stimuli, subsequently referred to as heat allodynia. Notably, although the perceived intensities were equated between the two skin conditions, heat allodynia yielded greater negative affect according to ratings subjects made using both a visual analog scale of 'unpleasantness' and a short form of the McGill pain questionnaire at the end of each scan. This result is consistent with the role of medial thalamic pathways in mediating affective and cognitive determinants of pain (Melzack and Casey, 1968; Price, 2000) and illustrates a close association of these responses with pain during pathological tissue states. It substantiates suggestions that different projection systems originating in the dorsal horn of the spinal cord mediate normal pain and pain during neurogenic inflammation (Hunt and Mantyh, 2001; Ikeda et al., 2003). In differentiating different pain types, the brain may engage different behavioral adaptations according to the physiological status of the body. Future studies may address the sensitivity of analgesic drugs in suppressing abnormal rather than normal nociceptive activity by using our capsaicin-heat model in combination with non-invasive neuroimaging such as PET or fMRI.

\section{References}

Andersson JL, Lilja A, Hartvig P, Langstrom B, Gordh T, Handwerker et al. Somatotopic organization along the central sulcus, for pain localization in humans, revealed by positron emission tomography. Exp Brain Res 1997;117:192-9.

Bingel U, Quante M, Knab R, Bromm B, Weiller C, Büchel C. Single trial fMRI reveals significant contralateral bias in response to laser pain within thalamus and somatosensory cortices. Neuroimage 2003; 18:740-8.

Casey KL. Forebrain mechanisms of nociception and pain: Analysis through imaging. Proc Natl Acad Sci USA 1999;96:7668-74.

Coghill RC, Sang CN, Maisog JM, Iadarola MJ. Pain intensity processing in the human brain: a bilateral, distributed mechanisms. J Neurophysiol 1999;82:1934-43.

Hunt SP, Mantyh PW. The molecular dynamics of pain control. Nat Neurosci 2001;2:83-91.

Ikeda H, Heinke B, Ruschewey R, Sandkühler J. Synaptic plasticity in spinal lamina I projection neurons that mediate hyperalgesia. Science 2003;299:1237-40.

Klein T, Magerl W, Hopf H-C, Sandkühler J, Treede R-D. Perceptual correlates of nociceptive long-term potentiation and long-term depression in humans. J Neurosci 2004;24:964-71.

Lorenz J, Cross DJ, Minoshima S, Morrow TJ, Paulson PE, Casey KL. A unique representation of heat allodynia in the human brain. Neuron 2002;35:383-93.

Melzack R, Casey KL. Sensory, motivational, and central control determinants of pain. In: Kenshalo DR, editor. Skin senses. Illinois, USA: Thomas Springfield; 1968. p. 423-43.

Ploghaus A, Tracey I, Gati JS, Clare S, Menon RS, Matthews PM, et al.. Dissociating pain from its anticipation in the human brain. Science 1999;284:1979-81.

Petrovic P, Kalso E, Petersson KM, Ingvar M. Placebo and opiod analgesia - imaging a shared neuronal network. Science 2002;295:1737-40.

Price DD. Psychological and neural mechanisms of the affective dimension of pain. Science 2000;288:1769-72.

Rainville P, Duncan GH, Price DD, Carrier B, Bushnell MC. Pain affect encoded in human anterior cingulate but not somatosensory cortex. Science 1997;277:968-71.

Sandkühler J, Ruscheweyh R. Opioids and central sensitisation: I. Preemptive analgesia. Eur J Pain, in press, doi:10.1016/j.ejpain. 2004.05.012.

Sprenger T, Berthele A, Platzer S, Boecker H, Tölle TR. What to learn from in vivo opioidergic brain imaging? Eur $\mathrm{J}$ Pain, in press, doi:10.1016/j.ejpain.2004.07.010. 